\title{
ON THE METAHEURISTIC OPTIMIZATION ALGORITHMS IN THE STRUGGLE FOR THE HOT FLOW CURVE APPROXIMATION ACCURACY
}

\author{
${ }^{1}$ Petr OPĚLA, ${ }^{1}$ Ivo SCHINDLER, ${ }^{1}$ Stanislav RUSZ, ${ }^{1}$ Vojtěch ŠEVČÁK, ${ }^{2}$ llija MAMUZIC \\ 1VSB - Technical University of Ostrava, Ostrava, Czech Republic, EU, petr.opela@vsb.cz \\ ${ }^{2}$ Faculty of Metallurgy, University of Zagreb, Sisak, Croatia
}

https://doi.org/10.37904/metal.2020.3466

\begin{abstract}
A hot flow curve approximation performed via flow stress models as well as artificial neural networks requires precisely estimated constants. This estimation is in the case of highly-nonlinear issues often solved via gradient optimization algorithms. Nevertheless, by natural processes or physical laws inspired approaches (metaheuristic algorithms) are also of high interest. In the submitted manuscript, three selected metaheuristic algorithms were compared under the approximation of an experimental hot flow curve dataset via the wellknown Hensel-Spittel relationship. One often used gradient algorithm was also included into this comparison. Results have showed that the metaheuristic algorithms are useful if such complex approximation model is applied and no estimate of material constants from a previous approximation issue is used. On the other hand, if this estimation exists, the gradient algorithms should provide a better solution.
\end{abstract}

Keywords: Hot-flow-curve approximation, genetic algorithm, artificial bee colony, fish swarm algorithm

\section{INTRODUCTION}

Since the last century, approximation of experimentally obtained sets of hot flow curves has been being realized via variously designed equations (flow stress models) - see e.g. overview in [1]. In the last years, however, so-called artificial neural network (ANN) approach [2] is gradually taking place instead of these models because of higher approximation performance. In addition, combination of the classical flow stress models with ANN approach is also studied [3]. Nevertheless, each mentioned method requires determination of approximation-model constants (synaptic weights and biases in the ANN terminology). It is clear that proper estimation of these constants plays a key role at achieving of high approximation accuracy. However, the ANN approach and some flow stress models represent a highly-nonlinear curve-fitting issues (practically without the possibility of rearrangement to linear form) which cannot be analytically solved by the Legendre's linear least squares method [4]. It is therefore understandable that these approximation issues are practically solvable with the use of numerical (iterative) techniques. For instance, gradient algorithms, e.g. Cauchy's gradient descent (GD) [5], Gauss-Newton (GN) [6] and especially Levenberg-Marquardt (LM) $[7,8]$ are by years proven and often used tools for the solving of nonlinear least squares problems. Nonetheless, besides of the mentioned gradient techniques, a promising alternative enabling to find an optimal solution has been being gradually developed since the mid-twentieth century - so-called metaheuristic algorithms are inspired by natural processes or physical laws [9]. These algorithms are advantageous because of their high robustness and the fact that an optimal solution can be found even despite of very rough initial estimation of approximationmodel constants. A large group of metaheuristic algorithms is represented by so-called population-based algorithms. This group namely includes e.g. Genetic Algorithm (GA), Fish Swarm Algorithm (FSA), Cat Swarm Optimization (CSO), Grey Wolf Optimization (GWO), Whale Optimization Algorithm (WOA) or Artificial Bee Colony $(A B C)$ and others - see overview in $[9,10]$. 
In the submitted manuscript, three representatives of the metaheuristic algorithms (GA, ABC and FSA) are compared with respect to the approximation accuracy of the well-known Hensel-Spittel relationship. In addition, the obtained results are subsequently compared with the commonly used gradient algorithm, namely Levenberg-Marquardt. The aim of this research is to compare the accuracy of proposed optimization algorithms and suitability of their use with respect to the hot flow curve approximation.

The research is performed on the experimental hot flow curve dataset of the chromium-molybdenum steel. This dataset has been obtained by means of the uniaxial hot compression tests on the Gleeble 3800 . The approximation procedure has been performed for the experimental curves under the following thermomechanical conditions: deformation temperatures $(1223 \mathrm{~K}, 1373 \mathrm{~K}$ and $1523 \mathrm{~K})$, strain rates $\left(0.1 \mathrm{~s}^{-1}\right.$, $1 \mathrm{~s}^{-1}, 10 \mathrm{~s}^{-1}$ and $100 \mathrm{~s}^{-1}$ ) and the true height strain up to 1.0 .

\section{CHARACTERIZATION OF THE CURRENT APPROXIMATION ISSUE}

The Hensel-Spittel (HS) relationship belongs among often-used equations which are intended to approximate an experimentally obtained hot flow curve dataset. The mathematical expression of the HS relationship used in this research is given by equation (1) [11]:

$\sigma(H S)=p_{1} \cdot \varepsilon^{p_{2}} \cdot \exp \left(\frac{p_{3}}{\varepsilon}\right) \cdot(1+\varepsilon)^{p_{4} \cdot T} \cdot \exp \left(p_{5} \cdot \varepsilon\right) \cdot \exp \left(p_{6} \cdot T\right) \cdot T^{p_{7}} \cdot \dot{\varepsilon}^{p_{8}} \cdot \dot{\varepsilon}^{p_{9} \cdot T}$

In equation (1), $\sigma(H S)(\mathrm{MPa})$ represents the calculated flow stress level and $\varepsilon(-)$ is the corresponding true strain value. The symbols of $T$ and $\varepsilon^{\prime}$ denote the deformation temperature $(\mathrm{K})$ and strain rate $\left(\mathrm{s}^{-1}\right)$, respectively. Model constants which have to be determined are then denoted as $p_{1}-p_{9}$.

In the present research, optimization procedure (i.e. searching for optimal values of $p_{1}-p_{9}$ ) was being realized with respect to the minimization of MSE (Mean Squared Error [12]) performance function:

$\min _{p} \operatorname{MSE}=\frac{1}{n} \cdot \sum_{i=1}^{n}\left[\sigma(H S)_{i}-\sigma(T)_{i}\right]^{2}$

In equation (2), $\sigma(H S)_{i}(\mathrm{MPa})$ represents an $i$-th value of the $\mathrm{HS}$-model output (equation (1)) and $\sigma(T)_{i}(\mathrm{MPa})$ is a corresponding target (experimental) value. Note, the $i=[1, n] \subset \mathbb{N}$, where $n$ is the number of datapoints of the examined flow curve dataset.

\section{METAHEURISTIC SEARCHING FOR OPTIMAL VALUES OF MODEL CONSTANTS}

The utilized metaheuristic optimization algorithms are schematically outlined in the Figure 1. The left side of the diagram shows mutual features of all metaheuristic algorithms. As the very first step, initial set of various solutions (vectors of model constants) is generated. This step is mostly performed pseudo-randomly. Nevertheless, solution from a previous approximation task can also be used, which can improve a convergence speed. As the second step, the produced solutions are evaluated with respect to the performance function equation (2) in this research. After this step, the predefined algorithm-terminating criteria are checked (e.g. minimum of performance function, number of iterations or elapsed time). If the terminating criteria are not met, the chosen metaheuristic algorithm is activated - see the right side of the diagram in Figure 1.

\subsection{Genetic Algorithm (GA)}

The genetic algorithm optimization has been established by Holland in 1975 [13] on the basis of Darwin's genetic principles and natural selection theory (1859) [14]. In accordance with the genetic terminology, constants of an approximation-model are labeled as genes. A specific combination of these genes (i.e. vector of constants) then represents an individual (i.e. possible solution). The genetic algorithm iteratively modifies the population of individuals through the production of new generations of possible solutions. Creation of new generation is performed by means of three mathematical processes which are analogy to the genetic principles: specifically, selection (choosing of appropriate individuals from the previous generation of 
solutions), crossover (i.e. exchange of genes between selected pairs of individuals (parents)) and mutation (random change of some genes). For more detailed description see e.g. [15].

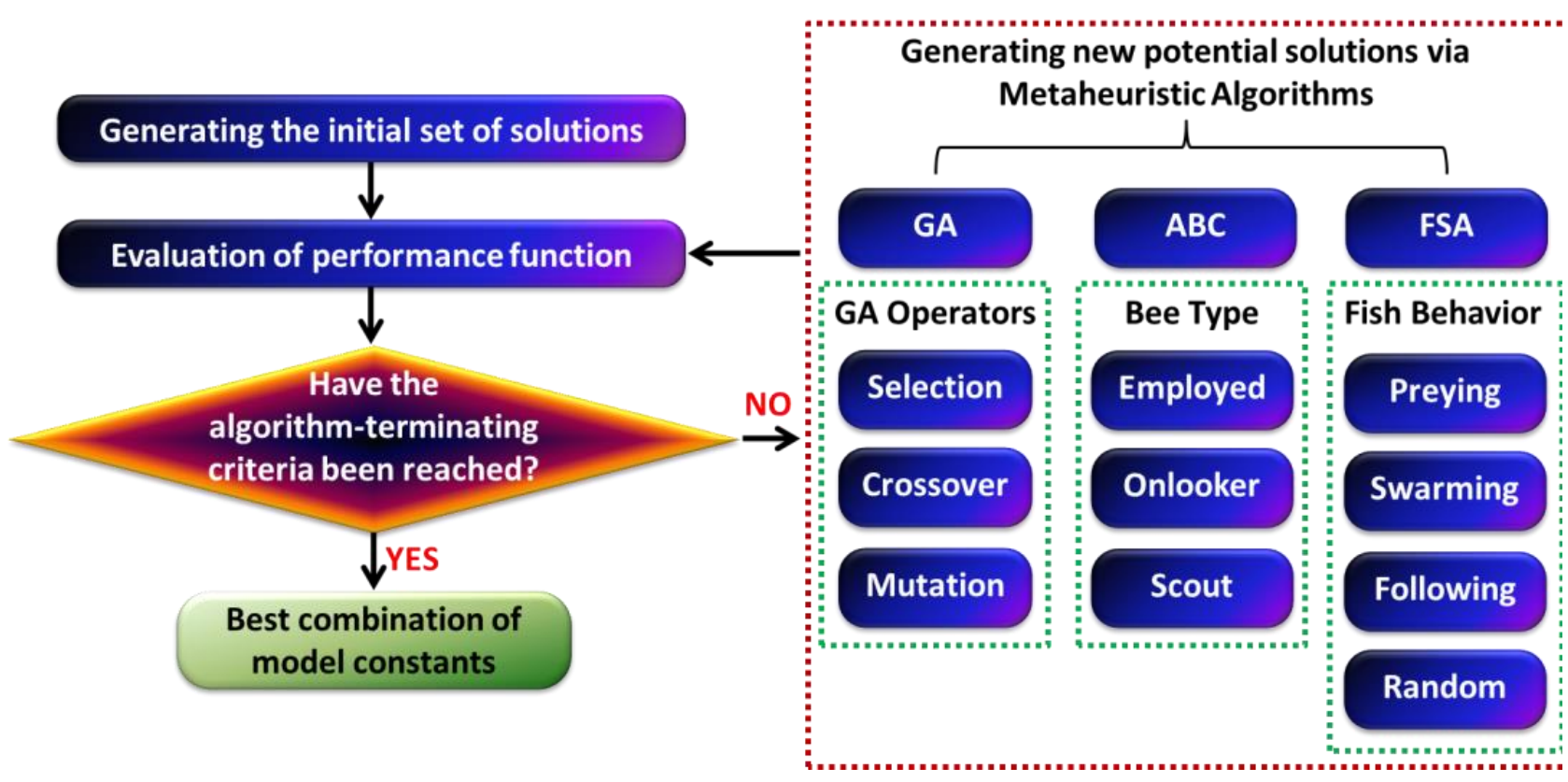

Figure 1 Basic block diagram of the utilized metaheuristic optimization algorithms

\subsection{Artificial Bee Colony $(A B C)$}

Based on the behavior of honeybee (Apis mellifera) swarm, Karaboga (2005) [16] has proposed the so-called artificial bee colony optimization. With respect to the specific terminology, vectors of possible solutions (i.e. constants of approximation model) are in the case of the $A B C$ algorithm entitled as food source positions. Similarly as in the case of the GA optimization, pseudo-randomly generated set of possible solutions (food source positions) is during the course of the $A B C$ algorithm gradually modified by the generating of new positions. The generating of new food source positions is mediated via two stages. In the first stage, so-called employed bees are sent to find new possible food sources in the neighborhood of the current sources when each employed bee explores the neighborhood of one source. Practically, each pseudo-randomly generated solution is modified in order to create a new one. If a newly calculated food source has a higher quality of nectar (i.e. better value of performance function), the previous source is replaced just by this new one. At the end of the first phase, nectar quality of each food source position is evaluated and submitted into the stage two. In the second stage, employed bees share their information about nectar quality of individual food sources with so-called onlooker bees in the hive. Each onlooker bee probabilistically selects one previously discovered food source position on the basis of roulette wheel selection. Onlooker bees then become to be employed bees and the $A B C$ algorithm is returned back to the stage one. It should be noted, the neighborhood of each food source position is explored by a predefined number of trials. A specific position is abandoned if its trial number is reached (i.e. if the employed bee cannot find better food source position). If this situation occurs, the employed bee become to be a scout bee and is sent to find a new potential food source (i.e. a new position is pseudo-randomly generated in order to replace the abandoned one). Detailed description and corresponding mathematical equations for food source generating can be found e.g. in [16,17].

\subsection{Fish Swarm Algorithm (FSA)}

The FSA algorithm has been invented by Li et al in 2002 [18] on the basis of imitation of fish swarm behavior at food searching. As in the case of the $A B C$ algorithm, pseudo-randomly generated set of possible solutions (food source positions) is gradually modified by the generating of new positions. Each artificial fish is 
associated with one source and searches for new sources in its neighborhood on the basis of four different types of behavior - see Figure 1. Each fish behavior type mediates the creation of new food source position by a different way. It can be said, the essence of this algorithm is very similar to the ABC algorithm. However, the mathematical approach for a new-food-source generating is, of course, different - see detailed overview in [10].

\section{RESULTS AND DISCUSSION}

The material constants $\left(p_{1}-p_{9}\right)$ of the Hensel-Spittel relationship (equation (1)) have been determined on the basis of each above introduced metaheuristic optimization algorithm. Their final values are sorted in Table 1. This table also contains the values of constants which were calculated on the basis of commonly used Levenberg-Marquardt (LM) gradient algorithm [7,8]. All utilized algorithms have been written and applied in the programing environment of the Octave-4.2.1 (free version of the well-known Matlab software).

Table 1 The values of the material constants of the Hensel-Spittel relationship

\begin{tabular}{|r|c|c|c|c|c|c|c|c|c|}
\hline & $p_{1}$ & $p_{2}$ & $p_{3}$ & $p_{4}$ & $p_{5}$ & $p_{6}$ & $p_{7}$ & $p_{8}$ & $p_{9}$ \\
\hline GA & $1.4 \cdot 10^{+3}$ & $1.2 \cdot 10^{-1}$ & $-6.3 \cdot 10^{-3}$ & $-1.1 \cdot 10^{-4}$ & $-1.6 \cdot 10^{-1}$ & $-2.9 \cdot 10^{-3}$ & $2.3 \cdot 10^{-1}$ & $-9.3 \cdot 10^{-2}$ & $1.5 \cdot 10^{-4}$ \\
\hline $\mathrm{ABC}$ & $2.7 \cdot 10^{+3}$ & $3.1 \cdot 10^{-2}$ & $-1.3 \cdot 10^{-2}$ & $3.3 \cdot 10^{-5}$ & $-1.0 \cdot 10^{-1}$ & $-3.6 \cdot 10^{-3}$ & $2.4 \cdot 10^{-1}$ & $-2.4 \cdot 10^{-1}$ & $2.6 \cdot 10^{-4}$ \\
\hline $\mathrm{FSA}$ & $1.5 \cdot 10^{+0}$ & $8.8 \cdot 10^{-2}$ & $-6.8 \cdot 10^{-3}$ & $1.1 \cdot 10^{-4}$ & $-2.8 \cdot 10^{-1}$ & $-4.7 \cdot 10^{-3}$ & $1.5 \cdot 10^{+0}$ & $-2.9 \cdot 10^{-1}$ & $3.0 \cdot 10^{-4}$ \\
\hline $\mathrm{LM}$ & $1.2 \cdot 10^{+0}$ & $1.1 \cdot 10^{-1}$ & $-5.2 \cdot 10^{-3}$ & $1.2 \cdot 10^{-5}$ & $-2.4 \cdot 10^{-1}$ & $-4.7 \cdot 10^{-3}$ & $1.5 \cdot 10^{+0}$ & $-2.9 \cdot 10^{-1}$ & $3.0 \cdot 10^{-4}$ \\
\hline
\end{tabular}

Figure 2 displays the graphical comparison among the experimental (boxes) and via the Hensel-Spittel relationship approximated (lines) flow curves. It can be said, with respect to the utilized metaheuristic optimization algorithms, the FSA approach (solid lines) gives the best approximation fit (practically visible at lower strain rates). The worst approximation is then given by the GA optimization (dash-dot lines). However, the representative of gradient algorithms (i.e. LM) gives practically the same results as the metaheuristic FSA optimization - the dot lines (LM) are superimposed by the solid lines (FSA). This is also confirmed by the similar values in their vectors of constants (see Table 1). It is also clear, the ABC algorithm (dash lines) provides similar results like the FSA and LM approaches. In order to quantify the approximation accuracy of the utilized algorithms, the relative percentage error, $\eta(\%)$, has been applied [3]:

$\eta_{i}=\frac{\sigma(H S)_{i}-\sigma(T)_{i}}{\sigma(T)_{i}} \cdot 100$

The distribution of the $\eta$-values is clearly expressed in the form of clustered histogram - see Figure 3, where each optimization algorithm is associated with a unique color. This histogram confirms the assumption about the close similarity of results which have been calculated by the FSA and LM algorithms - see the blue and black columns. The $\eta$-deviation of both approaches is ranging in the same range (i.e. from - $12 \%$ to $18 \%$ ) and the associated mean values, $\mu(\%)$, and standard deviations, $\sigma(\%)$, are almost identical. The ABC optimization (red columns) provides a less accurate approximation fit - its $\eta$-deviation is ranging in the wider range (from $-12 \%$ to $30 \%$ ), and the corresponding $\mu$-values and $\sigma$-values are in the comparison with the FSA or LM algorithms less favorable. With regard to the GA optimization approach (green columns), the $\eta$ values are ranging in the very wide range (from - $18 \%$ to $54 \%$ ), which is in a good accordance with the flow curve description in Figure 2. This fact is also reflected by the very unfavorable values of $\mu$ and $\sigma$.

It can be said, based on the obtained results, the FSA and LM algorithms are both for the optimization with regard to the Hensel-Spittel equation ideal candidates. However, it has to be said, the LM algorithm has not been able to calculate the constants of the HS relationship without an estimate from a previous approximation task. Gradient-based (i.e. derivative-based) nature of the LM algorithm is its stumbling-block since a derivative cannot be calculated if the approximating model returns error values - which can be occurred if the initial 
estimate of material constants is generated without considering the mathematical essence of mathematically complex model. In the case of the FSA algorithm, on the other hand, the final HS-model constants have been gained even with a rough pseudo-random estimate. It can be concluded, based on the obtained findings, the LM algorithm is more favorable if a previous estimation is available. The FSA algorithm should be then more useful in the case of new (mathematically complex) model implementation when the first estimation can be performed via the FSA algorithm and the final one via the LM approach.
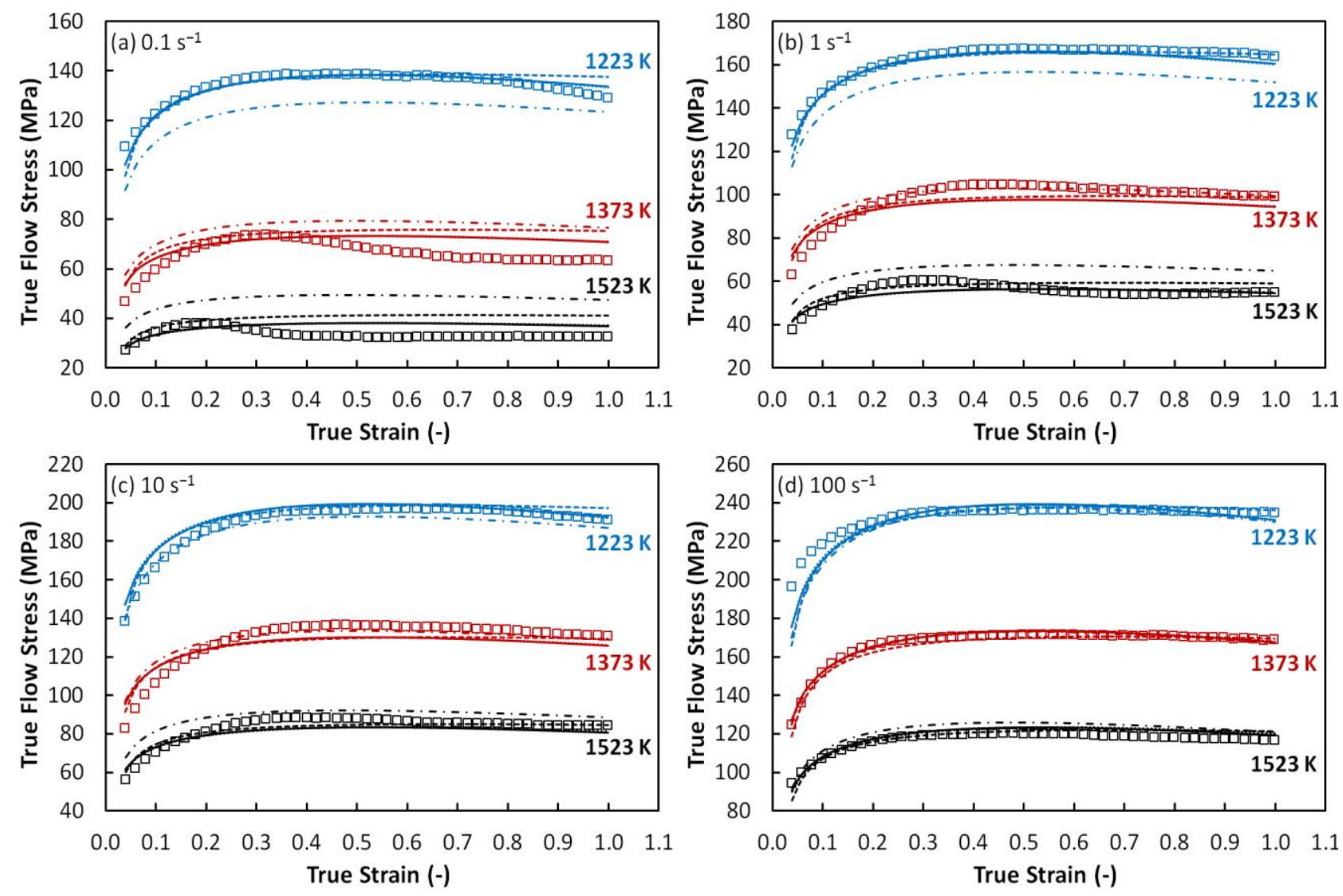

Figure 2 Flow curves of the chromium-molybdenum steel: experiment (boxes), calculated by Hensel-

Spittel: FSA (solid lines), ABC (dash lines), GA (dash-dot lines), LM (dot lines)

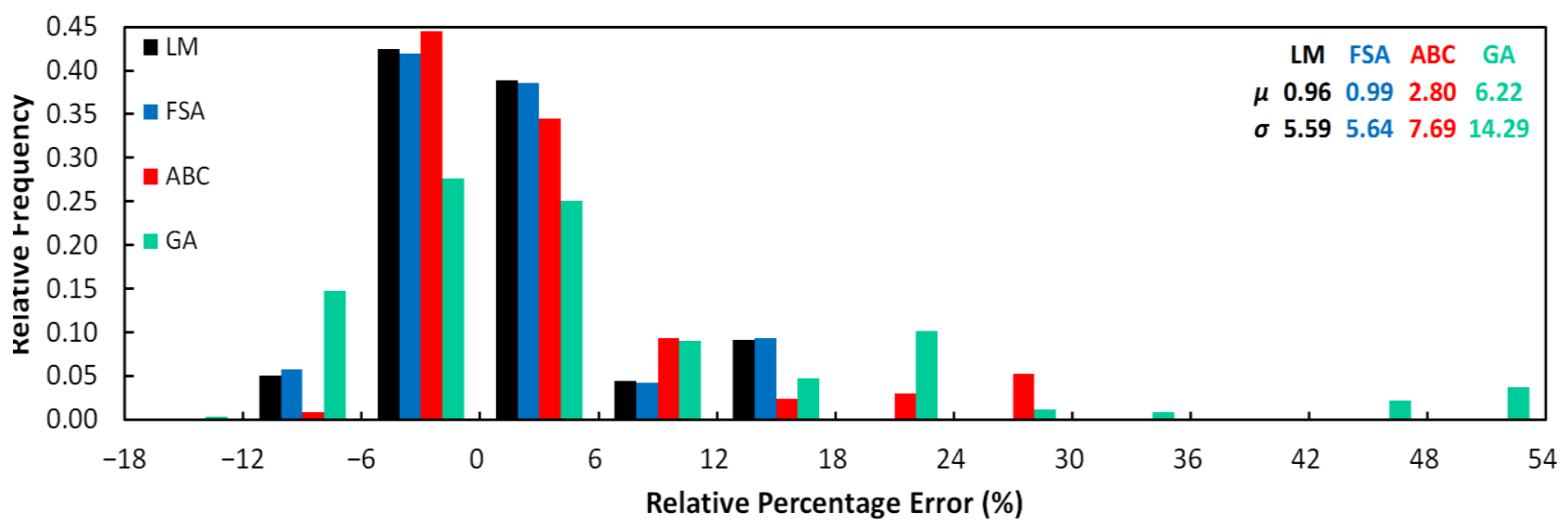

Figure 3 Distribution of the relative percentage error 


\section{CONCLUSION}

Three different metaheuristic optimization algorithms have been utilized and compared in the sense of struggle for the hot flow curve approximation accuracy. The submitted research has been performed on chromiummolybdenum steel. The well-known Hensel-Spittel model has been used as the approximation equation. Metaheuristic approaches, namely Genetic Algorithm (GA), Artificial Bee Colony (ABC) and Fish Swarm Algorithm (FSA), were applied to calculate the material constants of the selected approximation model. In addition, standardly used Levenberg-Marquardt (LM) optimization algorithm was employed for comparison with the proposed metaheuristic algorithms. The results have showed a high performance in the case of the FSA and LM. The LM algorithm has provided slightly better results - but only with the use of estimate from a previous approximation task. The FSA approach has been able to gain early the same results regardless of this previous estimate. Combination of both algorithms is thus recommended if a previous estimate is not available and approximation model is highly complex and sensitive to errors due to the roughly estimated constants.

\section{ACKNOWLEDGEMENTS}

The article was created thanks to the project No. CZ.02.1.01/0.0/0.0/17_049/0008399 (ČR and EU financial funds) and the Student Grant Competitions SP2020/88 and SP2020/39 (MŠMT ČR).

\section{REFERENCES}

[1] GRONOSTAJSKI, Z. The constitutive equations for FEM analysis. Journal of Materials Processing Technology. 2000, vol. 106, no. 1-3, pp. 40-44.

[2] McCULLOCH, W.S., PITTS, W.H. A Logical Calculus of Ideas Immanent in Nervous Activity. The bulletin of mathematical biophysics. 1943, vol. 5, no. 4, pp. 115-133.

[3] OPĚLA, P., SCHINDLER, I., KAWULOK, P., KAWULOK, R., RUSZ, S., RODAK, K. Hot Flow Curve Description of CuFe2 Alloy via Different Artificial Neural Network Approaches. Journal of Materials Engineering and Performance. 2019, vol. 28, no. 8, pp. 4863-4870.

[4] LEGENDRE, A.M. Nouvelles méthodes pour la détermination des orbites des comètes [New Methods for the Determination of the Orbits of Comets]. Mesnil-sur-l'Estrée: F. Didot, 1805.

[5] CAUCHY, A.L. Méthode générale pour la résolution des systèmes d'équations simultanées [General method for solving systems of simultaneous equations]. Comptes rendus hebdomadaires des séances de l'Académie des sciences. 1847, T25, pp. 536-538.

[6] GAUSS, J.C.F. Theoria motus corporum coelestium in sectionibus conicis solem ambientium [Theory of the Motion of the Heavenly Bodies Moving about the Sun in Conic Sections]. Hamburg: Friedrich Perthes and I. H. Besser, 1809.

[7] LEVENBERG, K. A Method for the Solution of Certain Non-Linear Problems in Least Squares. Quarterly of Applied Mathematics.1944, vol. 2, no. 2, pp. 164-168.

[8] MARQUARDT, D.W. An Algorithm for Least-Squares Estimation of Nonlinear Parameters. Journal of the Society for Industrial and Applied Mathematics. 1963, vol. 11, no. 2, pp. 431-441.

[9] YANG, X.S. Nature-Inspired Metaheuristic Algorithms. $2^{\text {nd }}$ edition. Cambridge: Luniver Press, 2010.

[10] DARWISH, A. Bio-inspired computing: Algorithms review, deep analysis, and the scope of applications. Future Computing and Informatics Journal. 2018, vol. 3, no. 2, pp. 231-246.

[11] HENSEL, A., SPITTEL, T. Kraft - und Arbeitsbedarf bildsamer Formgebungsverfahren. $1^{\text {st }}$ edition. Leipzig: Deutscher Verlag für Grundstoffindustrie, 1978.

[12] GAUSS, J.C.F. Theoria Combinationis Observationum Erroribus Minimis Obnoxiae [Theory of the Combination of Observations Least Subject to Errors]. Göttingen: Henricum Dieterich, 1823.

[13] HOLLAND, J.H. Adaptation in Natural and Artificial Systems. Michigan: University of Michigan Press, 1975. 
[14] DARWIN, Ch. On the Origin of Species by Means of Natural Selection, or the Preservation of Favoured Races in the Struggle for Life. London: John Murray, Albemarle Street, 1859.

[15] MITCHELL, M. An Introduction to Genetic Algorithms. Cambridge: The MIT Press, 1998.

[16] KARABOGA, D. An Idea Based on Honey Bee Swarm for Numerical Optimization. Technical report, 2005 [online]. [viewed 2019-12-14]. Available from: http://www.dmi.unict.it/mpavone/nc-cs/materiale/tr06_2005.pdf.

[17] KARABOGA, D. and BASTURK, B. A Powerful and Efficient Algorithm for Numerical Function Optimization: Artificial Bee Colony (ABC) Algorithm. Journal of Global Optimization. 2007, vol. 39, no. 3, pp. 459-471.

[18] LI, X., SHAO, Z. and QIAN, J. An Optimizing Method Based on Autonomous Animals: Fish-Swarm Algorithm. Systems Engineering Theory \& Practice. 2002, vol. 22, pp.32-38. 